\title{
Update in perioperative cardiac medicine 2021
}

\section{ABSTRACT}

Several studies published in the last year have shed light on the preoperative assessment of perioperative cardiovascular risk and on the need for anticoagulation in patients with postoperative atrial fibrillation, which are reviewed here.

\section{KEY POINTS}

The American University of Beirut HAS2 Index and the Updated Cardiac Risk Score are new, simple-to-use riskstratification tools for patients undergoing various types of surgical procedures.

Incorporating the Duke Activity Status Index into the preoperative evaluation helps identify patients at intermediate to high risk who may need modifications in perioperative care.

Another method of assessing exercise capacity is simply to ask patients if they can walk up 2 flights of stairs.

Preoperative measurement of N-terminal pro-brain natriuretic peptide also adds accuracy to the preoperative assessment, but the optimal cutoff value remains under study.

A study in patients with new-onset postoperative atrial fibrillation questions the efficacy of anticoagulation for preventing strokes while highlighting the bleeding risk.
C He Choice of CAlculators to predict 1 perioperative cardiac risk, assessment of a patient's exercise capacity, role of preoperative biomarkers, and need for anticoagulation after postoperative atrial fibrillation are controversial topics in perioperative medicine, and the evidence continues to evolve. This update will highlight new information in these areas based on publications from the past year.

\section{CARDIAC RISK CALCULATORS}

Current guidelines ${ }^{1}$ recommend the use of either the Revised Cardiac Risk Index, Myocardial Infarction or Cardiac Arrest Calculator, or American College of Surgeons Surgical Risk Calculator to estimate the risk of postoperative cardiac complications. Other calculators designed specifically for geriatric patients or for vascular surgery are also available. Most of these tools were developed retrospectively from databases or have not been externally validated.

\section{What's new? Two new calculators}

Two new prospectively developed calculators were recently reported, the American University of Beirut (AUB)-HAS2 Index ${ }^{2-4}$ and the Updated Cardiac Risk Score (UCRS). ${ }^{5}$

\section{The AUB-HAS2 Index}

Dakik et $\mathrm{al}^{2}$ prospectively derived the AUBHAS2 Index from 3,284 adult patients undergoing noncardiac surgery at the American University of Beirut Medical Center and validated it in 1,167,414 patients from the American College of Surgeons National Surgical Quality Improvement Program database. ${ }^{2}$ The primary outcome included death, myocardial infarction, or stroke at 30 days after surgery. The index and "HAS2" acronym are based on the following risk factors: 
- History of heart disease

- Symptoms of heart disease (angina or dyspnea)

- Age 75 or older

- Anemia (hemoglobin $<12$ mg/dL)

- Vascular surgery

- Emergency surgery.

The index stratifies risk in patients undergoing noncardiac surgery into 3 groups:

- Low risk (score 0-1)

- Intermediate risk (score 2-3)

- High risk (score $>3$ ).

The AUB-HAS2 Index was subsequently validated in a prospective cohort of $1,918 \mathrm{pa}$ tients at the American University of Beirut, although the primary outcome occurred in only 13 patients $(0.7 \%){ }^{3}$

Using $1,167,278$ patients from the $\mathrm{Na}$ tional Surgical Quality Improvement Program database, the performance of the AUB-HAS2 index was studied in 9 surgical specialty groups and in 8 commonly performed site-specific surgeries, compared with the Revised Cardiac Risk Index using receiver operating characteristic curves, and was superior overall (area under the curve 0.818 vs $0.716, P<.001$ ) as well as in all surgical subgroups (areas under the curve ranged from 0.71 in vascular and thoracic surgery to $>0.80$ in orthopedic, general, and plastic surgery). Additionally, it identified a large low-risk group of patients $($ score $=0$ ) and a high-risk group ( score $\geq 3$ ) with complication rates less than $1 \%$ and more than $10 \%$, respectively. ${ }^{4}$

Of note, there were differences between the AUB-HAS2 and Revised Cardiac Risk Index, limiting a true direct comparison but potentially enhancing applicability towards the overall population undergoing noncardiac surgery. The former included emergency surgery as well as some low-risk procedures, and the outcomes studied included stroke and allcause mortality at 30 days, which were not included in the Revised Cardiac Risk Index. ${ }^{4}$

\section{The Updated Cardiac Risk Score}

Scorcu et $\mathrm{al}^{5}$ derived the UCRS from 4,600 patients age 40 or older and validated it in another 2,735 patients in the Preoperative Assessment of Cardiovascular Risk in Patients Undergoing Elective Noncardiac Surgery, an observational prospective cohort study in Italy.
Outcomes included cardiovascular death, cardiac arrest, acute myocardial infarction, acute heart failure, atrioventricular block requiring cardiac pacing, and stroke within 30 days after surgery.

Four variables were significantly associated with the risk of a major perioperative cardiovascular event:

- High-risk surgery

- Preoperative glomerular filtration rate less than $30 \mathrm{~mL} / \mathrm{min} / 1.73 \mathrm{~m}^{2}$

- Age 75 or older

- History of heart failure.

Four risk classes were created, and their corresponding risks of a major cardiovascular complication were:

- $0.8 \%$ (95\% confidence interval [CI] 0.5-1.7)

- $2.5 \%$ (95\% CI 1.6-5.6)

- $8.7 \%$ (95\% CI 5.2-18.9)

- $27.2 \%$ (95\% CI 11.8-50.3).

The areas under the curve were higher for the UCRS than the Revised Cardiac Risk Index in both the derivation cohort $(0.86$ vs 0.79 ) and the validation cohort (0.77 vs 0.72 ). This study evaluated a wider range of complications than did the Revised Cardiac Risk Index, but like the Revised Cardiac Risk Index, it was a single-institution study and used creatine kinase-MB, not troponin, for postoperative surveillance. Once again, a true direct comparison is limited.

In summary, the AUB-HAS2 and UCRS are new, simple-to-use tools that accurately stratified risk in patients undergoing various types of surgical procedures. External validation is awaited using high-sensitivity troponin for postoperative surveillance.

\section{EXERCISE CAPACITY}

Estimating a patient's exercise capacity is part of the American College of Cardiology perioperative guideline algorithm, ${ }^{1}$ which suggests using at least 4 metabolic equivalents (METs) as a cutoff for adequate exercise capacity. Historically, this was done by asking patients how many blocks they could walk or flights of stairs they could climb, to determine their activity level.

However, the Measurement of Exercise Tolerance Before Surgery (METS) study ${ }^{6}$ demonstrated that a clinician's subjective assess-

\section{Two new risk indexes performed better than the Revised Cardiac Risk Index}


ment had a low discriminative ability to define poor exercise capacity compared with the gold standard of cardiopulmonary exercise testing, and it did not correlate well with postoperative cardiac complications. Instead, the study suggested using a more objective method, the Duke Activity Status Index score, ${ }^{7}$ which was more predictive of these complications.

\section{Duke Activity Status Index improves preoperative evaluation}

Wijeysundera et al, ${ }^{8}$ in a follow-up METS substudy, evaluated the ability of a specific cutoff value of the Duke Activity Status Index to predict complications after noncardiac surgery. In this nested cohort analysis of $1,546 \mathrm{pa}$ tients age 40 and older from the METS study, ${ }^{6}$ a score of 34 was identified as a threshold for distinguishing patients at risk for myocardial injury, myocardial infarction, moderate-to-severe complications, and new disability. However, although $97 \%$ of patients with scores of 35 or higher were deemed to have moderate or good functional capacity, only 15\% with scores of 34 or lower were judged as having poor functional capacity.

The authors ${ }^{8}$ urged caution in converting a A cutoff Duke Activity Status index of 34 was suggested, but 25 may be acceptable score to a specific number of METs because in this study, a Duke Activity Status Index score of 34 corresponded to $5 \mathrm{METs}$, whereas the recommended conversion formula in a nonsurgical setting yields 7 METs.

Fleisher, ${ }^{9}$ in a subsequent editorial, suggested that if a 50\% increased rate of events is considered clinically as opposed to statistically significant (since the absolute number of complications was low), then a Duke Activity Status Index score of less than 25 points (approximately 4 METs) would be a second cutoff to identify the subgroup in whom further testing should be considered if it would change management.

One of the limitations noted was that there was a bias excluding high-risk patients due to the study's requirement for strenuous preoperative exercise, which may have resulted in a low rate of myocardial infarction and death (only 26 patients, $1.7 \%$ ). 8,9

Summary. Incorporation of Duke Activity Status Index scores into preoperative evaluation helps to improve the accurate identification of intermediate-to-high-risk patients who warrant modifications in perioperative care. Using a cutoff of 34 was suggested by the study, although 25 may be an acceptable alternative. ${ }^{8}$

\section{Can you climb 2 flights of stairs?}

Lurati Buse et $\mathrm{al}^{10}$ performed a predefined secondary analysis of self-reported ability to walk up 2 flights of stairs and perioperative cardiovascular complications in 4,560 consecutive patients age 65 and older, or age 45 and older with a history of coronary artery disease, peripheral arterial disease, or stroke undergoing in-patient noncardiac surgery in Switzerland.

The primary end point was a composite of cardiac death and cardiac events at 30 days. Secondary end points included the same composite at 1 year, all-cause mortality, and myocardial injury.

Inability to climb 2 flights of stairs was independently associated with major adverse cardiac events and all-cause mortality at both 30 days (hazard ratio 1.63, 95\% CI 1.23-2.15) and 1 year. The addition of functional capacity information to the Revised Cardiac Risk Index improved risk classification. Although the use of a binary cutoff of 2 flights, while not addressing the impact of other daily activities or applying a validated formal functional capacity assessment tool (like the Duke Activity Status Index) was a limitation, the estimation based on "cut-off" daily activities is used by the guidelines.

Summary. Compared with the METS study, ${ }^{6}$ this study ${ }^{10}$ was larger, included higherrisk patients, and confirmed the association of self-reported exercise capacity with major adverse cardiac events. Additional information on self-reported exercise capacity will be forthcoming later in 2021 with the publication of the METREPAIR study of more than 15,000 patients across Europe.

\section{PREOPERATIVE NATRIURETIC PEPTIDES}

Multiple small studies have suggested that elevated levels of the natriuretic peptides brain natriuretic peptide (BNP) and $\mathrm{N}$-terminalproBNP (NT-proBNP) before surgery are associated with postoperative cardiac complications, and that measuring these peptides may improve risk prediction over using the Revised Cardiac Risk Index alone.

Although the American College of Cardi- 
ology guidelines ${ }^{1}$ did not recommend obtaining preoperative biomarkers, the Canadian Cardiovascular Society guidelines ${ }^{11}$ did recommend them for patients with a baseline risk of $5 \%$, which included those age 65 and older, those age 45 and older with cardiovascular risk factors, and those with a Revised Cardiac Risk Index of 1 or more undergoing noncardiac surgery. They also recommended postoperative troponin surveillance in patients whose NT-proBNP level was $300 \mathrm{pg} / \mathrm{mL}$ or higher.

\section{What's new? \\ Lower NT-proBNP cutoff suggested}

In a large study, Duceppe et $\mathrm{a}^{12}$ evaluated the utility of measuring NT-proBNP in predicting postoperative cardiac complications and suggested a different cutoff value.

This nested substudy of the prospective cohort Vascular Events in Noncardiac Surgery Patients Cohort Evaluation study evaluated 10,402 adults age 45 and older to determine whether preoperative NT-proBNP had additional predictive value beyond the Revised Cardiac Risk Index.

The primary end point, a composite of myocardial injury after noncardiac surgery or vascular death within 30 days after surgery, occurred in $12.2 \%$, but a more rigid outcome of myocardial infarction or all-cause mortality occurred in only $4.3 \%$. Stratified by preoperative NT-proBNP values, the incidence of the primary outcome was as follows:

- $\quad 100-199 \mathrm{pg} / \mathrm{mL}, 12.3 \%$ (226 of 1,843 )

- $200-1,499 \mathrm{pg} / \mathrm{mL}, 20.8 \%$ (542 of 2,608)

- $\geq 1,500 \mathrm{pg} / \mathrm{mL}, 37.5 \%$ (223 of 595).

Adding NT-proBNP thresholds to clinical stratification using the Revised Cardiac Risk Index improved net absolute reclassification by $25.8 \%$. Preoperative NT-proBNP values were also statistically significantly associated with 30-day all-cause mortality.

Summary. Compared with the Canadian guideline ${ }^{11}$ threshold of $300 \mathrm{pg} / \mathrm{mL}$ and over to identify patients at higher risk, this study found that a threshold of $200 \mathrm{pg} / \mathrm{mL}$ and over was associated with a risk greater than $5 \%$, but this cutoff needs to be validated externally.

\section{ANTICOAGULATION FOR POSTOPERATIVE ATRIAL FIBRILLATION}

New-onset postoperative atrial fibrillation occurs most frequently after cardiac and thoracic surgery, followed by vascular and major general surgery. It is also more common in the elderly and patients with underlying atherosclerotic cardiovascular disease and is associated with an increased risk of stroke and death within 30 days after surgery. ${ }^{13}$

New-onset atrial fibrillation was previously thought to be transient and benign; however, recent systematic reviews and meta-analyses reported an increased long-term risk for atrial fibrillation recurrence as well as for cardiovascular and cerebrovascular events. ${ }^{13,15}$ Although most patients with postoperative atrial fibrillation persisting more than 48 hours tend to receive anticoagulation, it is unclear whether they or patients with transient postoperative atrial fibrillation require anticoagulation or for what duration. ${ }^{14,15}$ Unknown is the long-term risk of cerebrovascular events in these patients and whether they should be treated similarly to patients with chronic nonvalvular atrial fibrillation, as the benefit of preventing thromboembolism must be balanced against the risk of bleeding with anticoagulation.

\section{What's new?}

\section{Anticoagulation questioned}

A new study in patients with new-onset postoperative atrial fibrillation questions the efficacy of anticoagulation for preventing stroke while highlighting the bleeding risk. ${ }^{16}$

Because how to manage new-onset postoperative atrial fibrillation after noncardiac surgery is unclear, Elharram et $\mathrm{a}^{16}$ performed a retrospective cohort study of 22,007 patients in Quebec from 1990 through 2015 to determine the association between oral anticoagulation use and hospitalization or emergency department visits for thromboembolic events and major bleeding.

The 6,475 of 22,077 patients (29\%) started on oral anticoagulation (81\% warfarin, $19 \%$ direct oral anticoagulants) within 30 days of discharge had higher $\mathrm{CHA}_{2} \mathrm{DS}_{2}-\mathrm{VASc}$ scores and lower HAS-BLED scores than those not given oral anticoagulation. A thromboembolic event occurred in 1,099 of 22,007 patients $(5 \%)$, and anticoagulation use was not associated with a lower risk (adjusted hazard ratio 0.89; 95\% CI 0.73-1.07) and did not differ based on class of anticoagulant. 
A major bleeding event occurred in 3,250 patients (15\%). Bleeding risk correlated with HAS-BLED scores, and anticoagulation use was associated with a higher risk of bleeding (hazard ratio 1.14; 95\% CI 1.04-1.25). Bleeding risk was higher in those on warfarin vs direct oral anticoagulants and those who had undergone thoracic surgery vs noncardiothoracic surgery.

Summary. Despite the increased risk of cardiovascular and cerebrovascular events after postoperative atrial fibrillation, in this study it appears that the thromboembolic risk in surgical patients differs from those with chronic nonvalvular atrial fibrillation.

In contrast to a Danish study, ${ }^{17}$ the El-

\section{REFERENCES}

1. Fleisher LA, Fleischmann KE, Auerbach AD, et al. 2014 ACC/AHA guideline on perioperative cardiovascular evaluation and management of patients undergoing noncardiac surgery. J Am Coll Cardiol 2014; 64(22):e77e137. doi:10.1016/j.jacc.2014.07.944

2. Dakik HA, Chehab O, Eldirani M, et al. A new index for pre-operative cardiovascular evaluation. J Am Coll Cardiol 2019; 73(24):3067-3078. doi:10.1016/j.jacc.2019.04.023

3. Dakik HA, Eldirani M, Kaspar C, et al. Prospective validation of the AUB-HAS2 Cardiovascular Risk Index. Eur Heart J Qual Care Clin Outcomes 2020 Oct 5:qcaa077. doi:10.1093/ehjqcco/qcaa077.

Anticoagulation did not statistically significantly lower the risk of thromboembolism in postoperative atrial fibrillation
4. Dakik HA, Sbaity E, Msheik A, et al. AUB-HAS2 Cardiovascular Risk Index: performance in surgical subpopulations and comparison to the Revised Cardiac Risk Index. J Am Heart Assoc 2020; 9(10):e016228. doi:10.1161/JAHA.119.016228

5. Scorcu G, Pilleri A, Contu P, et al. Preoperative assessment of cardiovascular risk in patients undergoing noncardiac surgery: the Orion study. Monaldi Arch Chest Dis 2020; 90(1). doi:10.4081/monaldi.2020.1169

6. Wijeysundera DN, Pearse RM, Shulman MA, et al. METS study investigators. Assessment of functional capacity to predict complications before major non-cardiac surgery: an international, prospective cohort study. Lancet 2018; 391(10140):2631-2640. doi:10.1016/S0140-6736(18)31131-0

7. Hlatky MA, Boineau RE, Higginbotham MB, et al. A brief selfadministered questionnaire to determine functional capacity (The Duke Activity Status Index). Am J Cardiol 1989; 64(10):651-654. doi:10.1016/0002-9149(89)90496-7

8. Wijeysundera DN, Beattie WS, Hillis GS, et al. Integration of the Duke Activity Status Index into preoperative risk evaluation: a multicentre prospective cohort study. $\mathrm{Br} J$ Anaesthiol 2020; 124(3):261-270. doi:10.1016/j.bja.2019.11.025

9. Fleisher LA. Preoperative evaluation in 2020: does exercise capacity fit into decision-making? Br J Anaesthiol 2020; 125(3):224-226. doi:10.1016/j.bja.2020.05.053

10. Lurati Buse GAL, Puelacher C, Menosi Gualandro D, et al. BASEL-PMI Investigators. Association between selfreported functional capacity and major adverse cardiac haram study showed that oral anticoagulation did not reduce thromboembolic events but did increase major bleeding. Until further data are available, the risk of chronic anticoagulation in patients with new-onset postoperative atrial fibrillation may be warranted only in patients with a thromboembolic risk above $1.5 \%$, the cutoff suggested by the Canadian Cardiovascular Society guidelines, ${ }^{11}$ which in the Elharam study correlated with a $\mathrm{CHA}_{2} \mathrm{DS}_{2}-\mathrm{VASc}$ score of 4 or higher, as opposed to a score higher than 2 in patients with chronic nonvalvular atrial fibrillation.

\section{DISCLOSURES}

The author reports no relevant financial relationships which, in the context of their contributions, could be perceived as a potential conflict of interest.

events in patients at elevated risk undergoing noncardiac surgery: a prospective diagnostic cohort study. Br J Anaesthiol 2021; 126(1):102-110. doi:10.1016/j.bja.2020.08.041

11. Duceppe E, Parlow J, MacDonald P, et al. Canadian Cardiovascular Society guidelines on perioperative cardiac risk assessment and management for patients who undergo noncardiac surgery. Can J Cardiol 2017 33(1):17-32. doi:10.1016/j.cjca.2016.09.008

12. Duceppe E, Patel A, Chan MTV, et al. Preoperative Nterminal pro-B-type natriuretic peptide and cardiovascular events after noncardiac surgery: a cohort study. Ann Intern Med 2020; 172(2):96-104. doi:10.7326/M19-250

13. AlTurki A, Marafi M, Proietti R, et al. Major adverse cardiovascular events associated with postoperative atrial fibrillation after noncardiac surgery: a systematic review and meta-analysis. Circ Arrhythm Electrophysiol 2020; 13(1):e007437. doi:10.1161/CIRCEP.119.007437

14. Pollak PT, Mitchell LB. Anticoagulation therapy for patients with new-onset postoperative atrial fibrillation after noncardiac surgery: Must do, may do, or don't do? Can J Cardiol 2020 Oct 13:S0828-282X(20)31020-5. doi:10.1016/j.cjca.2020.10.004

15. Albini A, Malavasi VL, Vitolo M, et al. Long-term outcomes of postoperative atrial fibrillation following non cardiac surgery: a systematic review and metanalysis. Eur J Intern Med 2021 Jan 2:S0953-6205(20)30470-2. doi:10.1016/j.ejim.2020.12.018.

16. Elharram M, Samuel M, AlTurki A, et al. Anticoagulant use and the risk of thromboembolism and bleeding in postoperative atrial fibrillation after noncardiac surgery. Can J Cardiol 2020 Sep 4:S0828-282X(20)30959-4. doi:10.1016/j.cjca.2020.08.023

17. Butt JW, Olesen JB, Havers-Borgersen E, et al. Risk of thromboembolismn associated with atrial fibrillation following noncardiac surgery. J Am Coll Cardiol 2018; 72(17):2027-2036. doi:10.1016/j.jacc.2018.07.088

Address: Steven L. Cohn, MD, MACP, SFHM, University of Miami Miller School of Medicine, 1120 NW 14th Street, CRB1140, Miami, FL 33136; Scohn@med.miami.edu 


\section{Update in perioperative cardiac medicine 2021}

In the April 2021 issue, in Cohn SL. Update in perioperative cardiac medicine 2021. Cleve Clin J Med 2021; 88(4):216-220, doi:10.3949/ccjm.88a.21014, in the fi nal paragraph, the sentence, "Until further data are available, the risk of chronic atrial fi brillation in patients with new-onset postoperative atrial fi brillation may be warranted only in patients with a thromboembolic risk above $1.5 \%$..." should read as follows: "Until further data are available, the risk of chronic anticoagulation in patients with new-onset postoperative atrial fi brillation may be warranted only in patients with a thromboembolic risk above $1.5 \%$..." This has been corrected online. 